\title{
Comparative cytotoxic effects of methanol, ethanol and DMSO on human cancer cell lines
}

\author{
Sinh Truong Nguyen ${ }^{1,2,}{ }^{*}$, Huyen Thi-Lam Nguyen ${ }^{1}$, Kiet Dinh Truong ${ }^{3}$
}

${ }^{1}$ Stem Cell Institute, University of Science Ho Chi Minh City, Ho Chi Minh City, Vietnam

${ }^{2}$ Cancer Research Laboratory, University of Science Ho Chi Minh City, Ho Chi Minh City, Vietnam

${ }^{3}$ Medical Genetic Institute, Ho Chi Minh City, Vietnam

\section{Correspondence}

Sinh Truong Nguyen, Stem Cell Institute, University of Science Ho Chi Minh City, Ho Chi Minh City, Vietnam

Cancer Research Laboratory, University of Science Ho Chi Minh City, Ho Chi Minh City, Vietnam

Email: sinhnguyen@sci.edu.vn

\section{History}

- Received: June 012020

- Accepted: July 162020

- Published: July 292020

DOI : 10.15419/bmrat.v7i7.614

\section{Check for updates}

\section{Copyright}

(c) Biomedpress. This is an openaccess article distributed under the terms of the Creative Commons Attribution 4.0 International license.

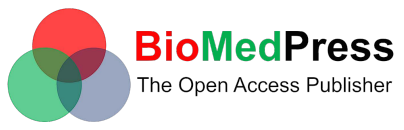

\begin{abstract}
Introduction: Anti-tumor drug screening is the most popular technique in drug discovery. In this technique, various agents were tested for their cytotoxicity on cell lines, particularly cancer cell lines. Since most of these agents are weakly soluble in water, they can be normally dissolved in lipophilic solvents. To obtain accurate results, the requirement of these solvents are that they be biocompatible and non-toxic to cells. The aim of this study was to investigate the biological effects of the three agents most commonly used as drug vehicles, i.e. dimethyl sulfoxide (DMSO), ethanol and methanol, using cell proliferation measurement techniques. Methods: To minimize the errors from the measurement techniques, this study used the xCelligence RTCA system which entails real-time monitoring of cell proliferation without dye labeling. Under the wide range concentration of solvents (from $0 \%$ to $100 \%$ of solvents by serial dilutions), the cell index (Cl) and slope of cell proliferation curves of HepG2, MDA-MB-231, MCF-7 and VNBRCA1 cell lines were analyzed (by the software provided by the xCelligence system). Results: The results showed that DMSO had a significant toxicity and inhibition of proliferation on 4 cell lines, at the concentrations of $10 \%, 5 \%$, $2.5 \%$ and $1.25 \%(p<0.0001)$. Methanol and ethanol inhibited cellular proliferation at the concentrations of $10 \%$ and $5 \%(p<0.0001)$. The concentrations of ethanol and methanol ranging from $2.5 \%$ to $0.15 \%$ concentration were well-tolerated by cells with respect to proliferation. Depending on the extracts or agents, each should be diluted in the suitable vehicle at the proper concentrations. Conclusion: Ethanol and methanol are good choices for solvents since they have low toxicity on HepG2, MDA-MB-231, MCF-7 and VNBRCA1 cell lines. However, in the case of agents only dissolvable in DMSO, low concentrations of DMSO from 0.6\%-0.015\% should be considered.
\end{abstract}

Key words: (Dimethyl sulfoxide) DMSO, ethanol, methanol, concentration, toxic, cancer cell

\section{INTRODUCTION}

In biological research, solvents for dilution of therapeutic drugs are necessary, especially for drugs that are weakly soluble in water. The most common solvents for use as vehicles for drug delivery purpose in biological studies are dimethyl sulfoxide (DMSO), ethanol and methanol. DMSO is an organic amphiphilic molecule that is widely used in cell biology; it exhibits a number of capabilities such as vasodilatory, diuretic, anti-inflammatory and bacteriostatic functions ${ }^{1,2}$.

In vitro, DMSO is routinely used for cryopreservation due to its high freeze temperature. However, its strong interaction with phospholipids makes it efficient in facilitating drug molecule delivery through the cell membrane, which is an area of study with great interest by many researchers. DMSO ranks high in terms of choice of solvent for drug delivery, despite its potential cytotoxicity on cells.

Alternative solvent that have been used in cell biology include ethanol and methanol. In drug screening, besides DMSO, crude plant extract could be dissolved in methanol or ethanol. However, ethanol could interfere with the structure of low cholesterol in the cell membrane and cause disorder of cellular physical activities $^{3}$. This damage in cell membrane could amplify the effect of therapeutic drugs, leading to nonreproducible results.

Furthermore, different cell types respond with different behavior to solvents ${ }^{4}$. Although solvent control could be used to compare to the drug efficacy in experiment, nonetheless if the solvents are toxic to the cells over treatment, the effect of the drug could be wrongly evaluated. It is important to evaluate and define the maximum concentration of the solvents that could be used for dissolving drugs in biological assays. Hence, it is necessary to understand the optimal concentration of the solvents on specific cell lines to ensure accurate and reproducible experimental results.

\section{MATERIAL - METHODS}

\section{Cell lines}

HepG2, MDA-MB-231 and MCF-7 cell lines was purchased from ATCC (Manassas, VA, USA). Cells was 
cultured in DMEM-F12 (Thermo Fisher Scientific, Waltham, MA, USA) complemented with 10\% FBS (Thermo Fisher Scientific) and $1 \%$ antibiotic (Thermo Fisher Scientific), and cultured in $5 \% \mathrm{CO}_{2}$ and $37^{\circ} \mathrm{C}$ incubator.

\section{Solvents}

Methanol, ethanol and DMSO was purchased from Merck (Darmstadt, Germany).

\section{Evaluation of label-free real-time cellular proliferation}

In brief, 2,000 cells were added into wells of E-plates (ACEA Biosciences, San Diego, CA, USA). After 24 hours of culture, cells were treated with DMSO, methanol and ethanol at the concentrations of $10 \%$, $5 \%, 2.5 \%, 1.25 \%, 0.6 \%, 0.3 \%, 0.15 \%$, and $0 \%$. The E-plates were then connected into the xCelligence RTCA machine (ACEA Biosciences) in the incubator for the next 48 hours. Cell proliferation was measured by recording the change in the impedance of electron flow caused by adherent cells. The experiments were done in triplicate $(n=3)$. The data were analyzed by the software attached to the xCelligence RTCA system (ACEA Biosciences).

\section{RESULTS}

After cells were cultured for 24 hours for cell attachment, the solvents were added; the proliferation curves were slightly perturbed (Figure 1). From that time point onward, the cell proliferation curves dispersed. From observation of the proliferation curves, when treated with methanol as the solvent, the red curve (concentration of 10\%) dipped down or became flattened in all the cell lines (Figure 1). Specially, the red curve (10\% methanol) for the VNBRCA1 cell line increased upwards; this demonstrated that the proliferation of VNBRCA1 was less affected even at the high concentration of methanol.

In contrast to methanol, DMSO induced more than one flattened curve in the 4 cell lines. For example, the red curve ( $10 \%$ concentration), light green curve (5\%), blue curve $(2.5 \%)$, and pink curve $(1.25 \%)$ all dipped downward.

Ethanol also appeared to induce divergent curves in the 4 cell lines. In HepG2, ethanol created 3 flattened curves compared to 2 curves in MDA-MB-231, MCF-7 and VNBRCA1. At the concentrations of 5\% and $2.5 \%$, ethanol inhibited the cell index (CI) values of the 3 cell lines MDA-MB-231, MCF-7 and VNBRCA1. However, the CI still showed upward increase at the concentration of $5 \%$ compared to that of $10 \%$ concentration for the MDA-MB-231, MCF7 and VNBRCA1 cell lines. The concentrations of $1.25 \%, 0.6 \%, 0.3 \%$, and $0.15 \%$ of ethanol induced upward curves in all 4 cell lines (Figure 1).

The results showed that DMSO significantly inhibited the proliferation of all the cell lines (HepG2, MDAMD-231, MCF-7 and VNBRCA1) at the concentrations of $10 \%, 5 \%, 2.5 \%$ and $1.25 \%$. The slope of the $\mathrm{CI}$ at these concentration were significantly different from that of the lower concentrations of $0.6 \%$, $0.3 \%$ and $0.15 \%(\mathrm{P}<0.0001)$ (Figure 2). Interestingly, DMSO at the concentration of $0.6 \%$ significantly affected cell growth of HepG2 and MCF-7, compared to the growth at the concentrations of $0.3 \%$ and $0.15 \%$. However, $0.6 \%$ DMSO did not affect the CI slope of MDA-MB-231 or VNBRCA1.

Ethanol strongly affected the cell growth of HepG2 cell line at the concentrations of $10 \%, 5 \%$ and $2.5 \%$. At the concentration of $2.5 \%$, ethanol had less effect on MCF-7, MDA-MB-231 and VNBRCA1, compared to HepG2. The CI slope of MCF-7, MDA-MB-231 and VNBRCA1 were positive values, compared to the negative slope value of HepG2 (Figure 2).

Methanol seemed to have the least impact on the cell lines in this study. Indeed, 10\% methanol showed a strong effect on all the cell lines while 5\% methanol significantly affected HepG2 cells and MCF-7 cells but not MDA-MB-231 or VNBRCA1 cells. Lower concentrations of methanol, such as $2.5 \%-0.15 \%$, showed no impact on any of the cell lines.

\section{DISCUSSION}

Although almost all solvents are toxic to cells in vitro, they are still necessary for dissolving drug agents for biological assays. It is essential to keep the concentration of the solvents at the most suitable concentration for biological experimentation. To do this, in this study, we compared the effects of three commonly used solvents (methanol, ethanol and DMSO) on 4 cancer cell lines (HepG2, MDA-MD-231, MCF-7 and VNBRCA1).

Some studies from the literature have stated that the critical concentration of DMSO should be $1 \%$, while others have used higher concentrations than that ${ }^{4}$. The question is what the proper concentration of DMSO for use should be while still being non-toxic on experimental cells.

In our study, the results showed that at high concentration of DMSO, such as $10 \%$ and $5 \%$, cell proliferation was strongly inhibited in all 4 cell lines. Interestingly, at the concentration $1.25 \%$ of DMSO, MDAMB-231 and MCF-7 cancer cells were still alive while 


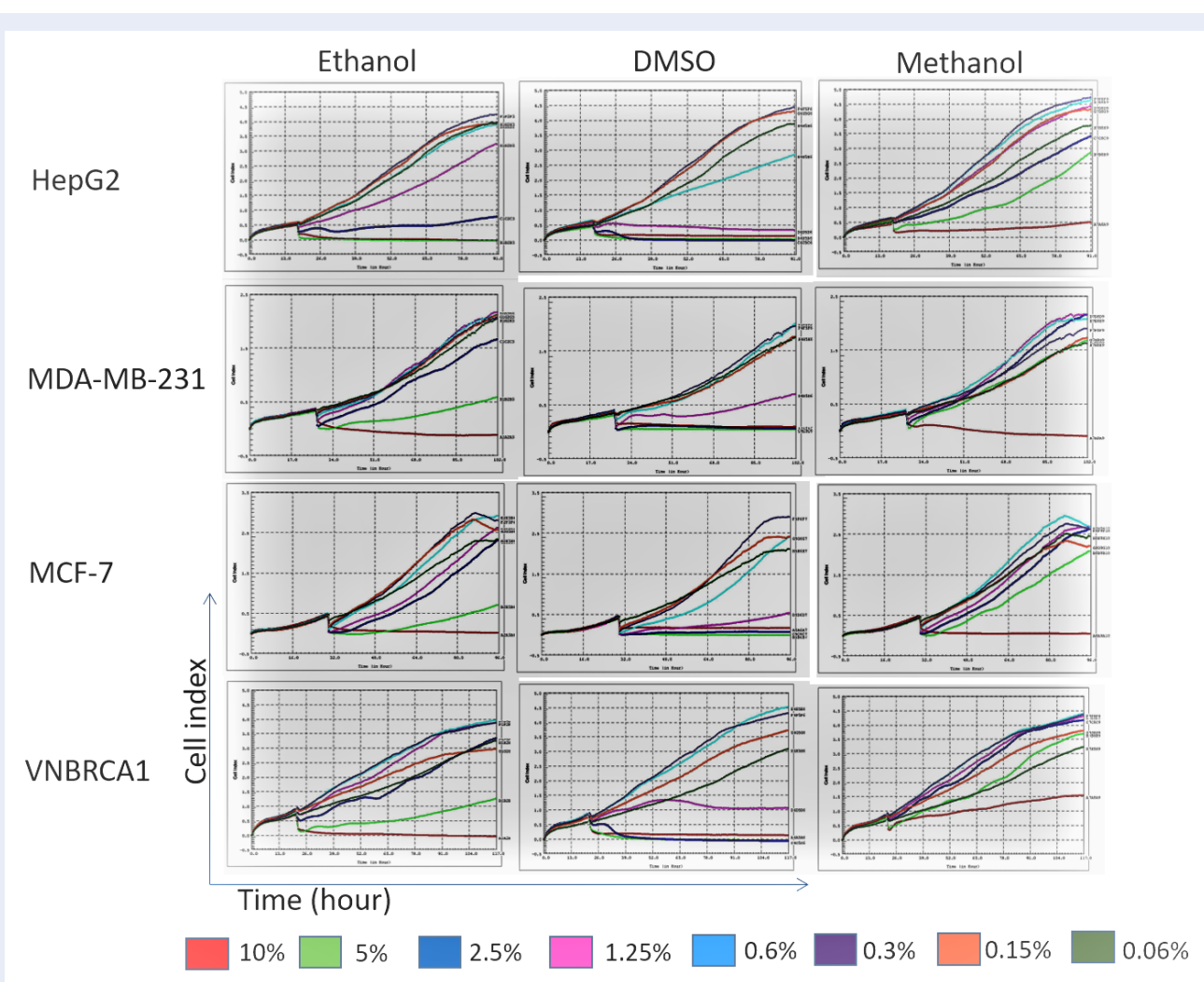

Figure 1: Cell index $(\mathrm{Cl})$ of cell lines when treated with solvents. Two thousand cells were added into the wells of E-plates. After 24 hours of culture, respective solvents were added at the concentrations of $10 \%, 5 \%$, $2.5 \%, 1.25 \%, 0.6 \%, 0.3 \%$, or $0.15 \%$. The column (y-axis) represents the Cl for the HepG2, MDA-MB-231, MCF-7 and VNBRCA1 cell lines. The row ( $x$-axis) represents the data for the solvents Ethanol, DMSO and Methanol over time. Cells were then cultured in the incubator for the next 3 days. The $\mathrm{Cl}$ was analyzed by the software of the xCELLigence RTCA system. The concentration range was coded by color, including $10 \%, 5 \%, 2.5 \%, 1.25 \%, 0.6 \%$, $0.3 \%, 0.15 \%$ and $0.06 \%$.

HepG2 cells showed a strong inhibition in proliferation. The sensibility to DMSO of different cell types may differ significantly. This also seems to be the case in other studies ${ }^{4,5}$. Therefore, it is essential to test the solvents at various concentrations if using different cell line models.

Previously, a study reported that lower concentrations of DMSO may lead to stimulation of proliferation of some cell types; for example, 0.05-0.2\% DMSO significantly increased the proliferation of RPMI- 8226 myeloma cells ${ }^{6}$. In the study herein, we did not recognize any significant difference in the doubling time of HepG2 and MDA-MB-231 at the concentrations of $0.3-0.15 \%$.

In this study, ethanol and methanol were demonstrated to be good solvent for use on HepG2, MDAMB-231, MCF-7 and VNBCRA1 cells. Ethanol is often used as a solvent for plant extracts and plantderived components ${ }^{7}$. There are toxicities to con- sider, but it is good for dissolution as long as it can dissolve the agents. Ethanol and methanol showed harmless effects on MDA-MB-231, MCF-7 and VNBRCA1 even at the high concentration of up to $2 \%$. Similarly, though not using the same cell lines as in our study, a previous study also found that $2.8 \%$ ethanol did not affect the cell viability of RAW $264.7^{8}$. In another report studying the effects of ethanol on HeLa cells, it was found that $5 \%$ ethanol (or higher concentrations) compromised cell viability ${ }^{9}$.

Interestingly, $5 \%$ methanol showed less toxicity on the 4 cell lines, while $5 \%$ ethanol significantly compromised all cell lines. A previous study showed that butanol is even more toxic than ethanol ${ }^{10}$; they observed some differences in toxicity between methanol, ethanol and butanol. The toxicity increase may be due to its longer carbon chain that can further intercalate into membranes and cause breaks in hydrogen bonds between lipid tails. 


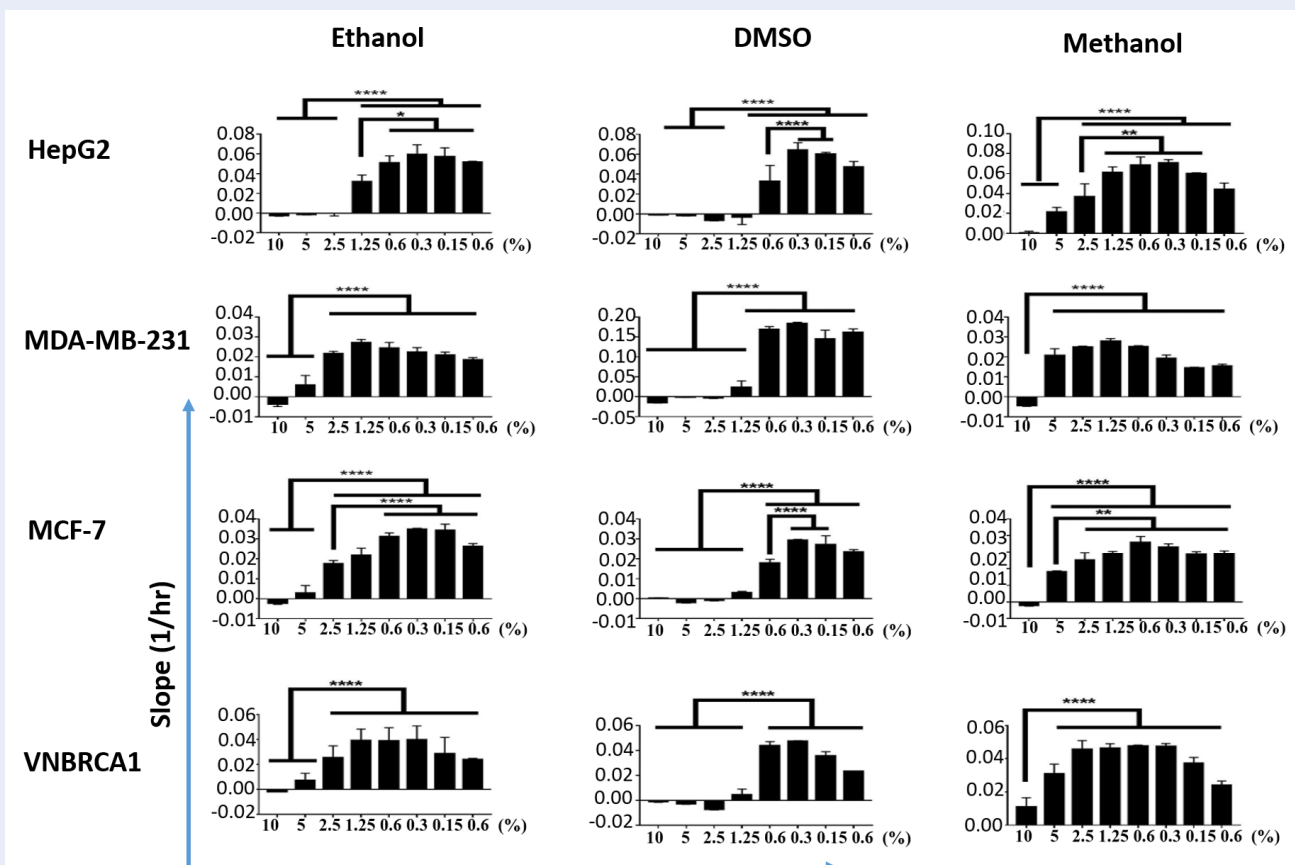

Concentration of solvents (\%)

Figure 2: The slope of the cell index $(\mathrm{CI})$ when treated with solvents. Cell lines used in this experiment were HepG2, MDA-MB-231, MCF-7 and VNBRCA1. Two thousand cells of each cell line were added into the wells of Eplates. After 24 hours of culture, Ethanol, DMSO and Methanol solvents were added, respectively, at the concentrations of $10 \%, 5 \%, 2.5 \%, 1.25 \%, 0.6 \%, 0.3 \%$, and $0.15 \%$ ( $0 \%$ as control). Cells were then cultured in the incubator for the next 3 days. The experiments was done in triplicate $(n=3)$. The slopes of the cell proliferation curves were calculated using the software of the $x$ Celligence system; ${ }^{* * *} P<0.0001$.

Ethanol is known to fluidize the cell membrane, leading to a disorder in trans-membrane protein flux such as that of $\mathrm{Mg} 2+{ }^{3,11}$. Ethanol can also inactivate ATPase and glycolytic enzymes, causing the inhibition of proliferation ${ }^{11,12}$. At the concentration of $2 \%, 1.25 \%$, $0.6 \%$ and $0.3 \%$, the effects of ethanol on cells was not significantly different; methanol also exhibited the same behavior as ethanol at those concentrations. If only some of the material dissolve in ethanol or methanol, that could be an advantage.

\section{CONCLUSION}

In biological studies, solvents are essential for dissolving agents that cannot be dissolved in water. Particularly, in cancer research, there are a lot of agents which require evaluation and testing on cell models. However, the toxicity of solvents on cancer cells is a problem. Hence, it is necessary to determine the most suitable concentrations to use in biological assays. Here, in this study, we found that DMSO should be used as a solvent in the range of concentration of $0.6 \%-0.15 \%$ on HepG2, MDA-MB-231, MCF-7 and
VNBRCA1 cells. Ethanol and methanol showed nontoxic effects on those cell lines at the concentrations of $1.25 \%-0.15 \%$. Some toxicity was tolerable when a control sample with solvent alone was used in experiments.

\section{ABBREVIATIONS}

DMSO: Dimethyl sulfoxide

\section{ACKNOWLEDGMENTS}

Not applicable.

\section{AUTHOR'S CONTRIBUTIONS}

All authors equally contributed in this study. All authors approved the final manuscript.

\section{FUNDING}

This work was supported by the Vietnam National University, Ho Chi Minh City, Vietnam, under grant A2015-18-01. 


\section{AVAILABILITY OF DATA AND MATERIALS}

Data and materials used and/or analysed during the current study are available from the corresponding author on reasionable request.

\section{ETHICS APPROVAL AND CONSENT TO PARTICIPATE}

Not applicable.

\section{CONSENT FOR PUBLICATION}

Not applicable.

\section{COMPETING INTERESTS}

The authors declare that they have no competing interests.

\section{REFERENCES}

1. Horita A, Weber LJ. Skin Penetrating Property of Drugs Dissolved in Dimethylsulfoxide (Dmso) and Other Vehicles. Life Sci. 1962;3:1389-1395. Available from: https://doi.org/10. 1016/0024-3205(64)90079-7.

2. Jacob SW, Bischel M, Herschler RJ. Dimethyl Sulfoxide (Dmso): A New Concept in Pharmacotherapy. Curr Ther Res Clin Exp. 1964;6:134-135.

3. Cartwright CP. Ethanol dissipates the proton-motive force across the plasma membrane of Saccharomyces cerevisiae. J Gen Microbiol. 1986;132:369-377. Available from: https: //doi.org/10.1099/00221287-132-2-369.
4. Timm $\mathrm{M}$, et al. Considerations regarding use of solvents in in vitro cell based assays. Cytotechnology. 2013;65(5):887894. PMID: 23328992. Available from: https://doi.org/10.1007/ s10616-012-9530-6.

5. Xing L, Remick DG. Mechanisms of dimethyl sulfoxide augmentation of IL-1 beta production. J Immunol. 2005;174(10):6195-6202. PMID: 15879116. Available from: https://doi.org/10.4049/jimmunol.174.10.6195.

6. Wen J, et al. Low Concentration DMSO Stimulates Cell Growth and In vitro Transformation of Human Multiple Myeloma Cells British. Journal of Medicine \& Medical Research. 2014;5(1):6574. Available from: https://doi.org/10.9734/BJMMR/2015/ 5276.

7. Liu Z. Preparation of botanical samples for biomedical research. Endocr Metab Immune Disord Drug Targets. 2008;8(2):112-121. PMID: 18537697. Available from: https: //doi.org/10.2174/187153008784534358.

8. Wakabayashi I, Negoro M. Mechanism of inhibitory action of ethanol on inducible nitric oxide synthesis in macrophages. Naunyn Schmiedebergs Arch Pharmacol. 2002;366(4):299 306. PMID: 12237742. Available from: https://doi.org/10.1007/ s00210-002-0625-z.

9. Forman S, et al. The effect of different solvents on the ATP/ADP content and growth properties of HeLa cells. J Biochem Mol Toxicol. 1999;13(1):11-15. Available from: https://doi.org/10. 1002/(SICI) 1099-0461(1999)13:1<11::AID-JBT2>3.0.CO;2-R.

10. Ly HV, Longo ML. The influence of short-chain alcohols on interfacial tension, mechanical properties, area/molecule, and permeability of fluid lipid bilayers. Biophys J. 2004;87(2):1013-1033. PMID: 15298907. Available from: https://doi.org/10.1529/biophysj.103.034280.

11. O IL. Adaptation of membrane lipids to alcohols. J Bacteriol. 1976;125:670-687. Available from: https://doi.org/10.1128/JB. 125.2.670-678.1976.

12. Ding J, et al. Tolerance and stress response to ethanol in the yeast Saccharomyces cerevisiae. Appl Microbiol Biotechnol. 2009;85(2):253-263. PMID: 19756577. Available from: https: //doi.org/10.1007/s00253-009-2223-1. 